\title{
Rupture of Round Bars by Tension
}

\section{T. Y. THOMAS}

1. Introduction. Let $\Sigma$ be a fixed surface in a solid. Suppose that an arbitrarily small slip of the material particles or discontinuity in the particle velocity, or its derivatives, develops over $\Sigma$ as a consequence of applied stress. It may then happen that this initially small discontinuity must necessarily increase indefinitely within a finite time, in view of the basic equations assumed to govern the behavior of the material. In such a case we shall say that $\Sigma$ is a surface of strong instability. Correspondingly, a moving surface $\Sigma(t)$, bearing a small discontinuity of the above type at time $t=0$, will yield a surface of strong instability $\Sigma(T)$ if the initial discontinuity becomes indefinitely large as $t \rightarrow T$. Again, a surface of strong instability $\Sigma^{*}$, distinct from the moving surface $\Sigma(t)$, may be generated by points on $\Sigma(t)$ at which the above discontinuity is infinite. Now it is our contention that fracture will occur over such surfaces as the physical manifestation of the inability of the material to support these infinite discontinuities. Hence we shall also refer to the surfaces of strong instability ${ }^{1}$ as surfaces of fracture or rupture.

It is commonly considered that fracture is produced as the result of the propagation of a $\operatorname{crack}^{2}$ in the solid. According to the present theory such a crack will move ${ }^{3}$ along a surface of instability or rupture, as above defined, on account of the inherent weakness of the medium over this surface.

It is natural, from the standpoint of this discussion, to consider the surface of separation $\Sigma$ of an elastic and plastic region in a solid as a possible surface of fracture since discontinuities in the velocity and stress, or their derivatives, must occur over such a surface, due to the differences in the character of the

${ }^{1}$ Discontinuities producing a weaker type of instability than the discontinuities considered in the definition of the surfaces of strong instability apparently do not result in rupture but may cause recognizable surface markings. See T. Y. Thomas, Plastic flow and fracture in solids, Jour. Math. and Mech., 7 (1958) pp. 291-322.

${ }^{2}$ For a comprehensive account of the theory of cracks reference may be made to the article on fracture by George R. Irwin, Encyclopedia of Physics, Vol. VII, Springer, Berlin, 1958.

${ }^{3}$ With regard to the velocity of propagation of the crack see A. A. WELLS \& D. Post, The dynamic stress distribution surrounding a running crack-A photographic analysis, Naval Research Laboratory, Washington, D. C., Report 4935, 1957. In this connection see also T. Y. Thomas, Plastic disturbances whose speed of propagation is less than the velocity of a shear wave, Jour. Math. and Mech., 7 (1958) pp. 893-900. 
equations governing the behavior of the material in the elastic and plastic states. In our previous article ${ }^{4}$ on fracture we confined ourselves exclusively to the treatment of such surfaces of separation. But this restriction is not necessary and we can also consider fracture surfaces within a purely plastic region, as we shall do in the present paper dealing with the problem of the rupture of round bars by tension. ${ }^{5}$

A uniform tension or load, if sufficiently large, applied at the ends of a bar in the direction of its axis will produce fracture in a plane perpendicular to the direction of the axis. Hence let us consider a plane $\Sigma$ perpendicular to the axis of the bar. We assume the material on one side of $\Sigma$ to be in the elastic state, on the other side in the plastic state. From the nature of this fracture problem we are justified in supposing that the plane $\Sigma$ is at rest, i.e. fixed relative to the dynamically allowable coordinate system employed, and that the material on the elastic side of $\Sigma$ is in a state of dynamical equilibrium. ${ }^{6}$ Our problem is now to ascertain if the magnitude of the singularity in the velocity of the flow over the plane $\Sigma$ must increase in the manner postulated to cause the rupture of the bar.

Since the discontinuity in the velocity and its derivatives on a surface $\Sigma$ plays a dominant role in this theory of fracture we may assume the continuity of the stress components and their derivatives, as well as the density and its derivatives, provided the conditions of the problem are such that this assumption does not unduly limit the possibility of discontinuities in the velocity and derivatives of the velocity. We have thus been led, in our treatment of the problem at hand, to consider that the stress and its first partial derivatives are continuous over the plane $\Sigma$. Utilizing, in addition, only the cylindrical symmetry of the flow in the plastic region, inherent in the nature of this problem, the following result has been proved (\$7). If the load on the bar is held at the yield point the magnitude of the velocity, induced initially on the plane $\Sigma$ by the plastic flow, will become indefinitely large within a finite time. Hence $\Sigma$ will be a plane of instability over which the bar will be subject to rupture, in accordance with the viewpoint of this theory. Incidently, it may be emphasized here that this result depends essentially on the tensile character of the applied load. For it is also shown in $\$ 7$ that any assumed initial velocity on the plane $\Sigma$, subject only to the symmetry requirements of this problem, will be damped out by a compressive force, conforming to the fact that a bar does not fracture over a plane perpendicular to its axis as a result of compression.

In view of the symmetrical character of the flow, it is natural to raise the

${ }^{4}$ Plastic flow and fracture in solids, loc. cit. pp. 291-322.

5 This investigation was conducted at the request of the Naval Research Laboratory and has been supported by the Office of Naval Research under Contract Nonr-908(09), Indiana University, NR 041037.

${ }^{6}$ Because of this condition of dynamical equilibrium we shall be concerned only with a static stress field in the elastic region. Hence the term "elastic" need not be restricted to the meaning of this word in the classical elasticity theory but can be interpreted in a broader sense. 
question if there will not be surfaces of revolution about the axis of the bar, other than the above plane $\Sigma$, over which rupture can occur. An investigation of this problem, under the assumption that the stress components and their first partial derivatives are continuous across the surface of rupture, shows that the only possibility is a circular cone whose generators make an angle of $35^{\circ} 16^{\prime}$ with a plane perpendicular to the axis of the bar (see \$8). When this cone is combined with the plane we obtain the well known "cup and cone" type of rupture sometimes experienced by round bars under tension.

In deriving the above results it is supposed that the material possesses a sharp yield point at which it passes abruptly from the elastic to the plastic state. Assuming the linear or classical theory of elasticity prior to the development of plastic flow, the complete stress-strain relationship is usually represented by a drawing such as that in Fig. 1, in which the straight line $A B$ shows the relation between the stress $\sigma$ and the strain $e$ of the linear elasticity theory and the part $B C$ indicates the behavior of the plastic material. Correspondingly, the rupture of the bar is represented by Fig. 2 . Now the actual behavior of a ductile material is shown more accurately by a stress-strain relationship such as we see in Fig. 3 with the part $A B$, which is approximately a straight line, representing the strictly elastic domain. When the stress in the bar reaches the upper part $C$ of this curve "necking" will occur, after which the bar may rupture

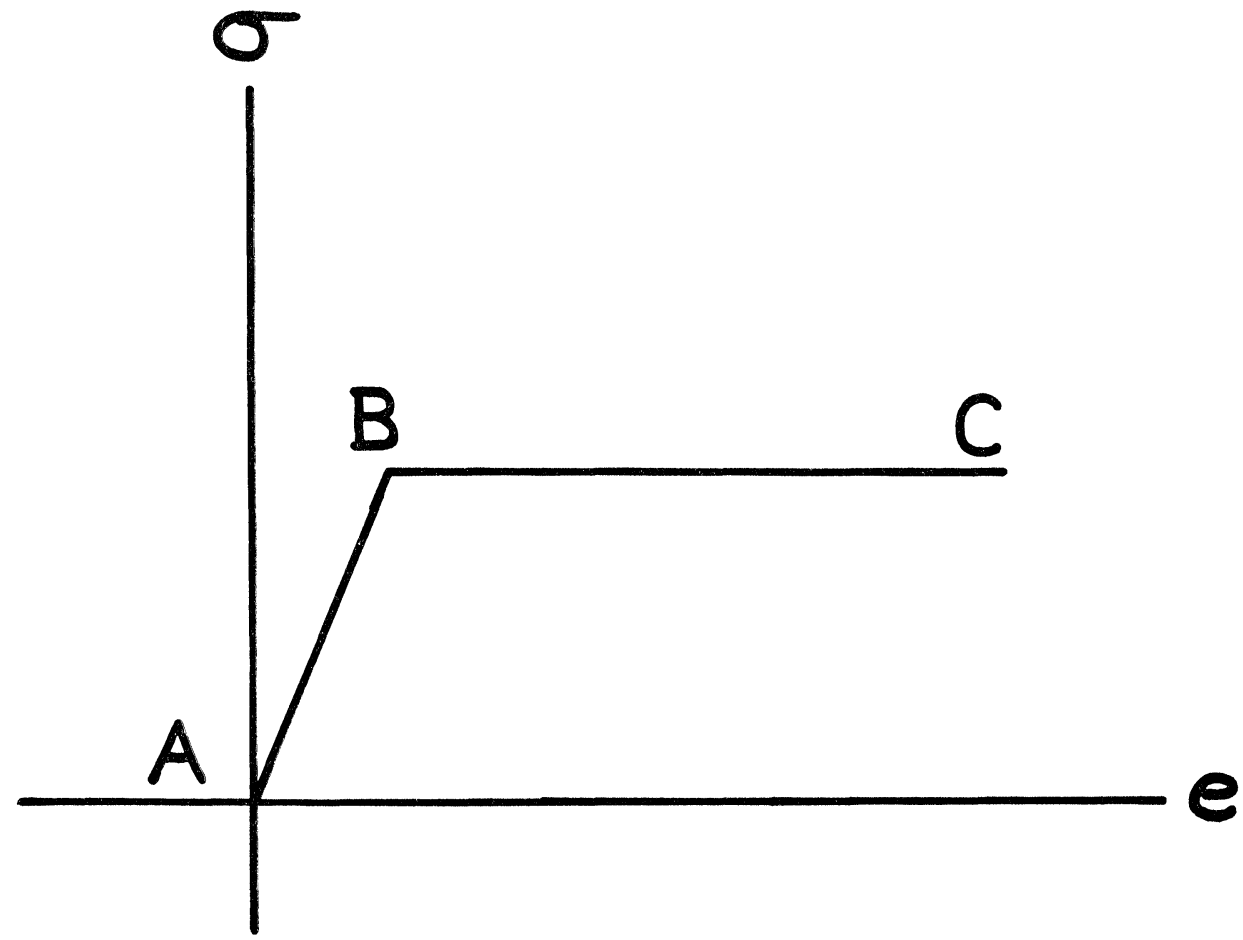

Figure 1. 


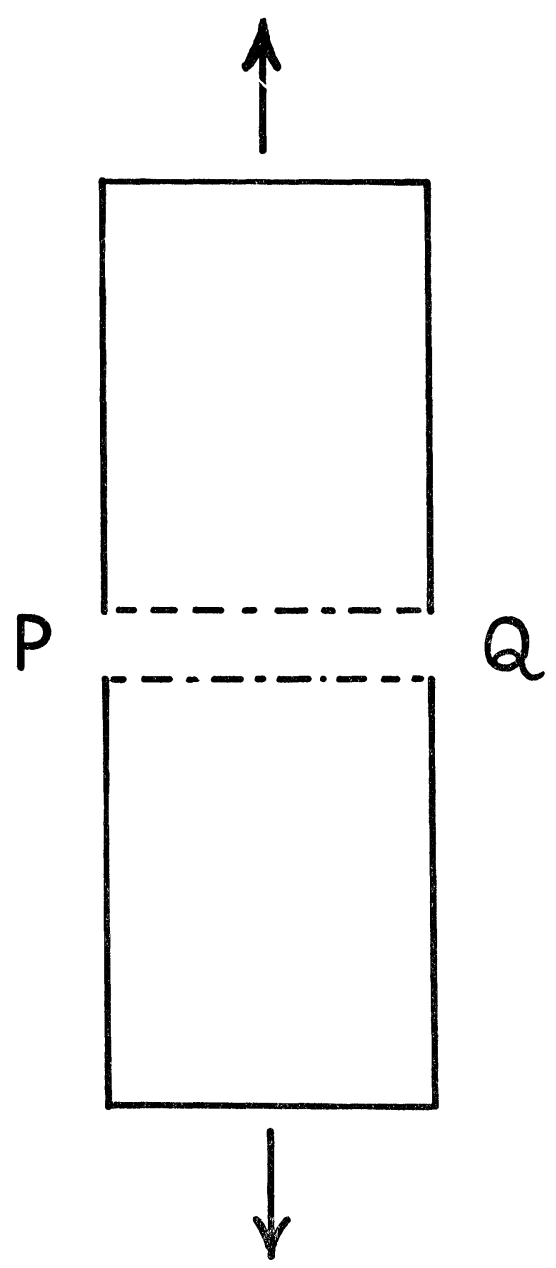

Figure 2.

as shown in Fig. 4. In the case of such ductile materials it would appear that plastic flow may be present in the minimum section $P Q$ of the neck, so that we are actually concerned with the development of rupture within an entirely plastic region. We have treated this problem in $\$ 9$ and have shown that rupture may occur over the plane of minimum section $P Q$, under the assumption that the density, the stress components and the first partial derivatives of these components are continuous across this section of the bar.

2. General form of the equations for plastic flow. In our previous articles we have referred the equations for the plastic flow of a solid to a system of rectangular coordinates. Denoting by $y^{i}$ the coordinates of this rectangular system, in which the equations are assumed valid, let us make an arbitrary 


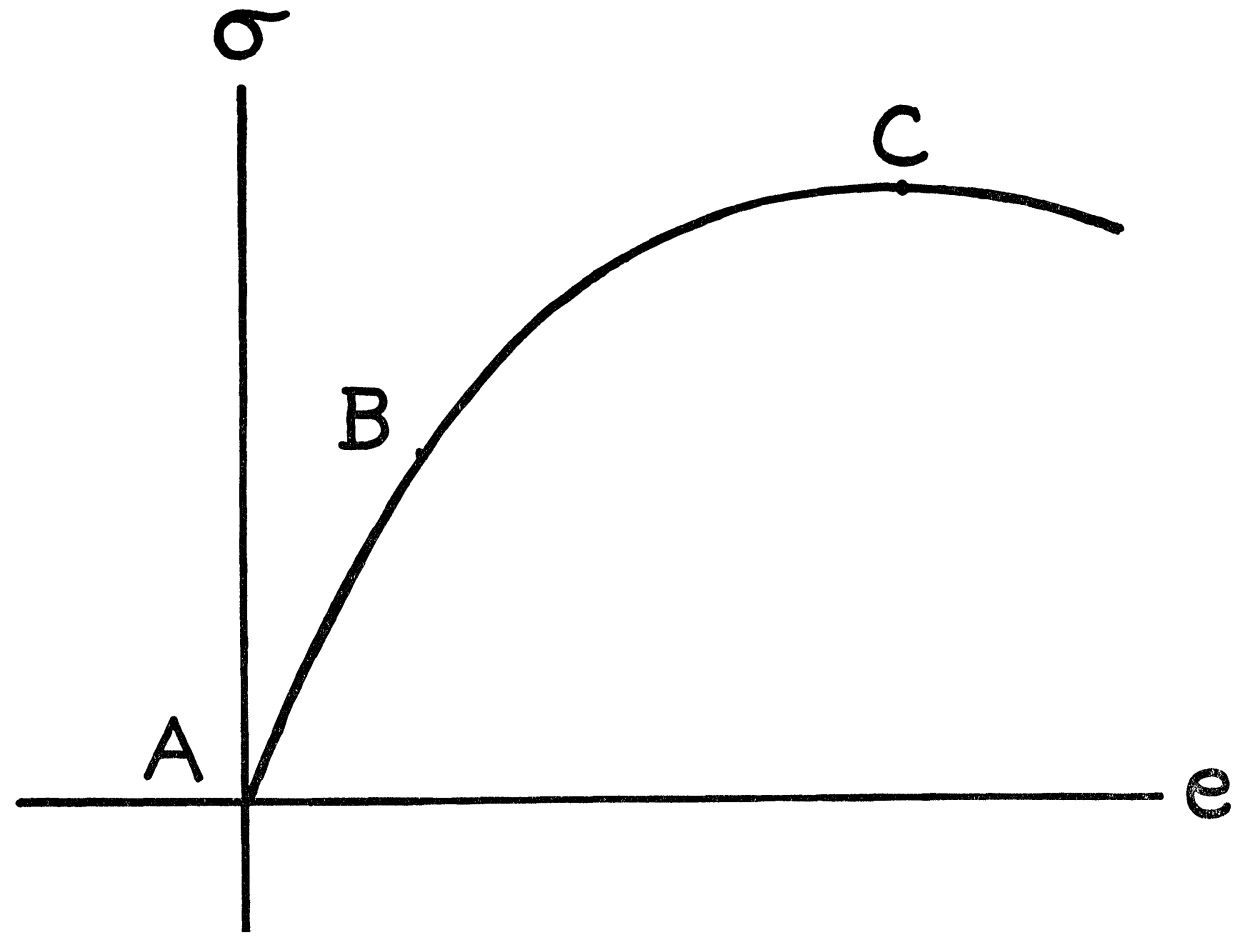

Figure 3.

differentiable transformation $x \leftrightarrow y$ independent of the time $t$. We shall now consider the form of the equations for plastic flow relative to the $x$ system.

Let us first observe that if $T_{i j}$ are the components of a tensor in the $x$ system the partial derivatives

$$
\frac{\partial T_{i j}}{\partial t}
$$

are the components of a tensor under coordinate transformations $x \leftrightarrow \bar{x}$ which do not involve the time $t$. Hence, under such transformations, the quantities $d T_{i j} / d t$, defined by

$$
\frac{d T_{i j}}{d t}=\frac{\partial T_{i j}}{\partial t}+T_{i j, k} v^{k}
$$

are tensor components, where the comma denotes covariant differentiation and the $v^{k}$ are the contravariant components of the velocity $v$. A summation is to be made on the index $k$ in the last term of (2.1), in accordance with the usual convention. The above quantities $d T_{i j} / d t$ are the components of the total time derivative of the tensor $T$. The total time derivative of a vector or a tensor of any order is defined in a corresponding manner.

We shall raise or lower indices on the symbol of the components of any tensor 


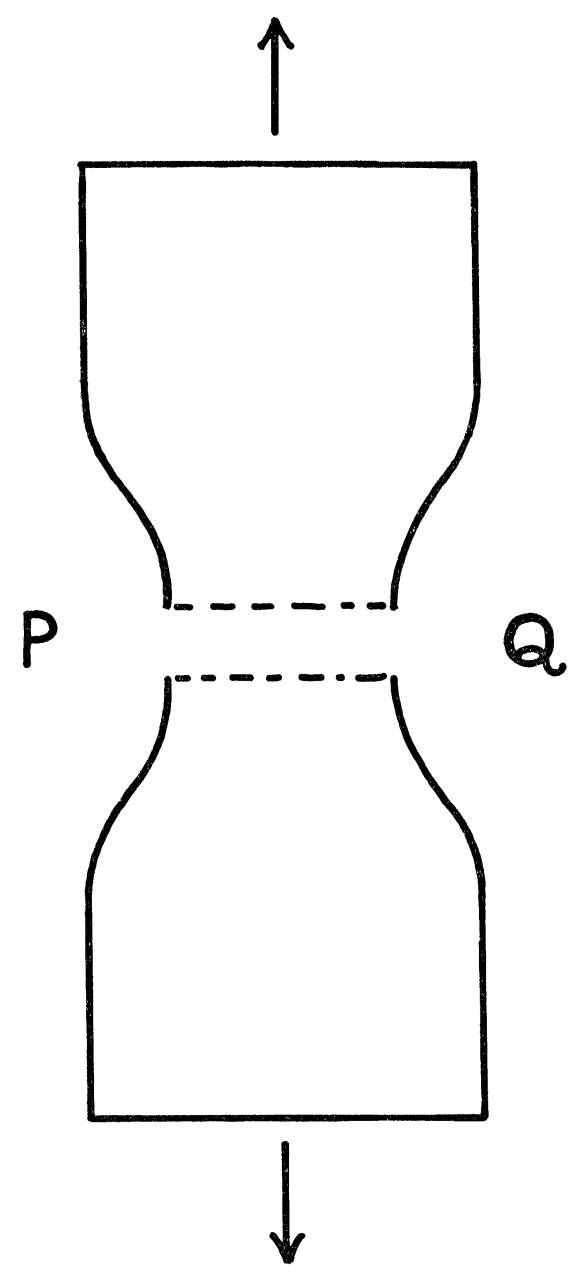

Figure 4.

by the usual procedure. Thus if $g_{i j}$ and $g^{i f}$ are the covariant and contravariant components of the metric tensor $g$ we have the following relations:

$$
v_{i}=g_{i j} v^{i} ; \quad v^{i}=g^{i j} v_{i}
$$

between the covariant components $v_{i}$ and the contravariant components $v^{i}$ of the velocity vector.

With these preliminary remarks the complete system of relations which we assume to govern the plastic flow can be written as follows:

$$
g^{i k} \sigma_{i j, k}=\rho \frac{d v_{i}}{d t}, \text { equations of motion, }
$$




$$
\begin{gathered}
\frac{\partial \rho}{\partial t}+\rho_{, i} v^{i}+\rho g^{i j} v_{i, j}=0, \quad \text { equation of continuity, } \\
g^{i j} v_{i, j}=0, \text { equation of incompressibility, } \\
\frac{d \sigma_{i j}^{*}}{d t}=2 \mu\left(\epsilon_{i j}-\psi \sigma_{i j}^{*}\right), \quad \text { Prendtl-Reuss equations, }
\end{gathered}
$$

where

$$
\psi=\frac{1}{K} g^{i k} g^{i m} \sigma_{i j}^{*} \epsilon_{k m} ; \quad K=g^{i k} g^{j m} \sigma_{i j}^{*} \sigma_{k m}^{*} .
$$

The above quantities $\mu$ and $K$ are material constants, and the second equation (2.7) is sometimes referred to as the von Mises or quadratic yield condition. Also the quantities $\epsilon_{i j}$ and $\sigma_{i j}$ are the components of the rate of strain tensor and the stress tensor respectively, while the $\sigma_{i j}^{*}$ are the components of the stress deviator. The tensors $\epsilon, \sigma^{*}$ and the rate of strain deviator $\epsilon^{*}$ are defined by

$$
\begin{aligned}
& \epsilon_{i j}=\frac{1}{2}\left(v_{i, i}+v_{j, i}\right), \\
& \sigma_{i j}^{*}=\sigma_{i j}-\frac{1}{3}\left(g^{a b} \sigma_{a b}\right) g_{i j}, \\
& \epsilon_{i j}^{*}=\epsilon_{i j}-\frac{1}{3}\left(g^{a b} \epsilon_{a b}\right) g_{i j} .
\end{aligned}
$$

It follows from (2.5), (2.8) and (2.10) that $\epsilon_{i j}^{*}=\epsilon_{i i}$, and hence we can omit the star on the components $\epsilon_{i j}^{*}$, as we have done in writing the equations (2.6) and the first equation (2.7). The components of the total time derivatives which appear in (2.3) and (2.6) are, of course, given by equations analogous to (2.1).

The relations $(2.3), \cdots,(2.7)$ are valid in the $x$ coordinate system as above defined. This follows since (1) the relations hold in the rectangular system $y$ by assumption and (2) they are invariant under differentiable transformations $x \leftrightarrow x$ which do not involve the time.

3. First order compatibility conditions in general coordinates. Let the surface $\Sigma$ be defined by

$$
x^{i}=x^{i}\left(u^{1}, u^{2}\right) \quad(i=1,2,3),
$$

where the $u$ 's are parametric coordinates of points of the surface. Then, obviously, the quantities

$$
x_{\alpha}^{i}=\frac{\partial x^{i}}{\partial u^{\alpha}} \quad(\alpha=1,2),
$$

are contravariant under transformations $x \leftrightarrow x$ of the $x$ coordinates (see $\$ 2$ ) and, correspondingly, are covariant under arbitrary differentiable transformations $u \leftrightarrow \bar{u}$ of the parametric coordinates; moreover, for $\alpha$ fixed the three quantities $x_{\alpha}^{i}$ are the components of a vector tangent to the surface $\Sigma$. In conformity with all the above equations, we shall now adopt the convention that 
Latin indices have the range 1, 2, 3 and Greek indices the range 1, 2. The summation convention will apply for Latin and Greek indices subject to these ranges.

If the surface $\Sigma$ is represented by an equation of the form $f(x, t)=0$ the covariant components of the unit normal vector $\nu$ to the surface are conveniently given by the equations

$$
\nu_{i}=\frac{\frac{\partial f}{\partial x^{i}}}{\sqrt{g^{j k} \frac{\partial f}{\partial x^{j}} \frac{\partial f}{\partial x^{k}}}} \cdot
$$

For the contravariant components of this normal vector we have

$$
\nu^{i}=g^{i i} \nu_{i} \text {. }
$$

We shall also have occasion to employ the fundamental metric tensor of the surface $\Sigma$ whose covariant and contravariant components will be denoted by $g_{\alpha \beta}$ and $g^{\alpha \beta}$ respectively. No confusion will result, in general, from this notation since the components of the metric tensors of the space and the surface $\Sigma$ can be distinguished from one another by means of their Latin and Greek indices. However, in dealing with individual components, e.g. the components $g_{11}, g_{12}$ and $g_{22}$, we must specify whether these are components of the metric tensor of the space or the surface $\Sigma$.

Denoting by $Z$, with indices omitted, any one of the components of a vector or tensor, let us define the surface quantities

$$
A=[Z] ; \quad B=\left[Z_{, i}\right] \nu^{i},
$$

where the bracket [ ] stands for the difference of the quantity enclosed at contiguous points on the two sides of the surface $\Sigma$ and the comma indicates covariant differentiation relative to the space coordinates $x$. It is seen immediately that the geometrical compatibility conditions of the first order for $Z$, relative to the general $x$ coordinate system of $\$ 2$, are given by

$$
\left[Z_{, j}\right]=B \nu_{j}+g^{\alpha \beta} g_{j k} A_{; a} x_{\beta}^{k},
$$

where the semicolon denotes covariant differentiation relative to the surface coordinates $u$, since these relations reduce to the proper compatibility conditions for the rectangular $y$ system and are, moreover, invariant under the transformation by which one passes from the $y$ to the $x$ system.

To illustrate the construction of the kinematical compatibility conditions of the first order relative to the $x$ system, let us consider the particular case of the covariant velocity vector. From the equations of transformation of the components $v_{k}$ of this vector it follows immediately that the discontinuities $\left[v_{k}\right]$ transform by the equations

$$
\left[\bar{v}_{i}\right]=\left[v_{k}\right] \frac{\partial x^{k}}{\partial \bar{x}^{i}}
$$


under the transformations $x \leftrightarrow \bar{x}$ of $\S 2$. Let us now equate the $\delta$ time derivatives, defined exactly as in our previous paper on compatibility conditions, ${ }^{7}$ of the two members of the equations (3.4). We thus obtain

$$
\frac{\delta\left[\bar{v}_{i}\right]}{\delta t}=\frac{\delta\left[v_{k}\right]}{\delta t} \frac{\partial x^{k}}{\partial \bar{x}^{i}}+\left[v_{k}\right] \frac{\partial^{2} x^{k}}{\partial \bar{x}^{i} \partial \bar{x}^{j}} \frac{\delta \bar{x}^{j}}{\delta t} .
$$

But

$$
\frac{\delta \bar{x}^{j}}{\delta t}=G \bar{\nu} j
$$

where $G$ is the coordinate velocity of the moving surface $\Sigma$ in the direction of its unit normal $\nu$. Obviously $G$ is an absolute scalar function on the surface $\Sigma$. Now

$$
\bar{\Gamma}_{i j}^{m} \frac{\partial x^{k}}{\partial \bar{x}^{m}}=\frac{\partial^{2} x^{k}}{\partial \bar{x}^{i} \partial \bar{x}^{i}}+\Gamma_{a b}^{k} \frac{\partial x^{a}}{\partial \bar{x}^{i}} \frac{\partial x^{b}}{\partial \bar{x}^{i}},
$$

where the $\Gamma$ 's and $\bar{\Gamma}$ 's are the Christoffel symbols in the $x$ and $\vec{x}$ systems respectively. Making the substitution (3.6) and eliminating the second derivatives in (3.5) by means of (3.7), we find that the resulting equations can be written

$$
\frac{\delta\left[\bar{v}_{i}\right]}{\delta t}-\left[\bar{v}_{m}\right] \bar{\Gamma}_{i j}^{m} G \bar{\nu} j=\left(\frac{\delta\left[v_{k}\right]}{\delta t}-\left[v_{m}\right] \Gamma_{k a}^{m} G \nu^{a}\right) \frac{\partial x^{k}}{\partial \bar{x}^{i}} .
$$

Putting

$$
\left[v_{k}\right]_{, t}=\frac{\delta\left[v_{k}\right]}{\delta t}-\left[v_{m}\right] \Gamma_{k a}^{m} G \nu^{a}
$$

and making a corresponding substitution for the quantities $\left[\bar{v}_{\boldsymbol{i}}\right]_{, t}$, we now have

$$
\left[\bar{v}_{i}\right]_{, t}=\left[v_{k}\right]_{, t} \frac{\partial x^{k}}{\partial \bar{x}^{i}}
$$

in place of (3.8). But (3.10) expresses the fact that the quantities $\left[v_{k}\right]_{, t}$ are vector components under transformations $x \leftrightarrow \bar{x}$ of the $x$ coordinates. Moreover, we observe that $\left[v_{k}\right]_{, t}$ reduces to the corresponding $\delta$ time derivative $\delta\left[v_{k}\right] / \delta t$ in the rectangular $y$ system.

Now consider the relations

$$
\left[\frac{\partial v_{i}}{\partial t}\right]=-G\left[v_{i, k}\right] \nu^{k}+\left[v_{i}\right]_{, t}
$$

which reduce to the kinematical conditions of compatibility for the vector $v$ in the rectangular $y$ system on account of the remark at the end of the preceding paragraph. These relations are invariant in form under transformations $x \leftrightarrow \bar{x}$

${ }^{7}$ Extended compatibility conditions for the study of surfaces of discontinuity in continuum mechanics, Jour. Math. and Mech., 6 (1957) pp. 311-322; also A correction to "Extended compatibility conditions for the study of surfaces of discontinuity in continuum mechanics", ibid., 6 (1957) pp. 907-908. No correction of any of the equations of the original article on extended compatibility conditions is necessary in case the normal velocity $G$ is constant in space. 
of the $x$ coordinates. This follows from (3.10) and the fact that the quantities $\left[\partial v_{i} / \partial t\right]$ and $\left[v_{i, k}\right] \nu^{k}$ enjoy corresponding equations of transformation. Hence (3.11) represents the kinematical conditions of compatibility for the velocity relative to the $x$ system.

Corresponding to the conditions (3.3), the kinematical conditions of compatibility of the first order can be written symbolically as

$$
\left[\frac{\partial Z}{\partial t}\right]=-G B+A_{, t} .
$$

The last term in this relation will be called the invariant time derivative of the quantity $A$ and its components will be given by a formula analogous to the above formula (3.9) for the components of the invariant time derivative of the discontinuity $\left[v_{k}\right]$ of the velocity vector. The conditions (3.12) are, of course, invariant under arbitrary differentiable transformations $x \leftrightarrow \bar{x}$ of the $x$ coordinates.

Remark. If the surface $\Sigma$ is stationary, i.e. if $G=0$, it follows from (3.9) and (3.11) that

$$
\left[\frac{\partial v_{i}}{\partial t}\right]=\frac{\delta\left[v_{k}\right]}{\delta t}
$$

But this relation is obviously an identity when $G=0$. Similarly, the general kinematical conditions of compatibility (3.12) are satisfied identically and hence can be disregarded in dealing with stationary surfaces $\Sigma$.

4. Cylindrical coordinates. We shall now select coordinate systems appropriate to the treatment of the round bar under tension. Thus the rectangular $y$ system will be chosen to have its $y^{3}$ axis along the axis of the bar; this places the $y^{1}$ and $y^{2}$ axes in a plane perpendicular to the axis of the bar. Cylindrical coordinates $r, \theta, z$ will now be defined by the following transformation:

$$
y^{1}=r \cos \theta ; \quad y^{2}=r \sin \theta ; \quad y^{3}=z .
$$

To avail ourselves of the general formulæ of the preceding sections we shall identify the coordinates $r, \theta, z$ with the coordinates $x^{1}, x^{2}, x^{3}$ respectively of the $x$ system.

The values of the covariant components $g_{i j}$ and the contravariant components $g^{i f}$ of the metric tensor for the cylindrical system are readily calculated. Expressed in matrix notation we have

$$
\left\|g_{i j}\right\|=\left\|\begin{array}{lll}
1 & 0 & 0 \\
0 & r^{2} & 0 \\
0 & 0 & 1
\end{array}\right\|
$$


and

$$
\left\|g^{i i}\right\|=\left\|\begin{array}{ccc}
1 & 0 & 0 \\
0 & \frac{1}{r^{2}} & 0 \\
0 & 0 & 1
\end{array}\right\|
$$

Specifically, the value of the component $g_{i j}$ or $g^{i j}$ is given by the element of the $i^{\text {th }}$ row and $j^{\text {th }}$ column of the matrix $\left\|g_{i j}\right\|$ or $\left\|g^{i j}\right\|$.

We shall also need to consider the Christoffel symbols $\Gamma_{j k}^{i}$ which are determined by the general formula

$$
\Gamma_{j k}^{i}=\frac{1}{2} g^{i m}\left(\frac{g_{m i}}{\partial x^{k}}+\frac{\partial g_{m k}}{\partial x^{i}}-\frac{\partial g_{i k}}{\partial x^{m}}\right) .
$$

Using this formula and the above values of the components of the metric tensor, we find that the Christoffel symbols are given in the cylindrical system by the equations

$$
\Gamma_{22}^{1}=-r ; \quad \Gamma_{12}^{2}=\frac{1}{r} ; \quad \Gamma_{j k}^{i}=0 \text { otherwise. }
$$

In this problem of the round bar acted upon by a uniform tension at its ends, the stress components $\sigma_{i j}$ and the velocity components $v_{i}$ are obviously independent of the angular coordinate $\theta$, i.e.

$$
\sigma_{i j}=\sigma_{i j}(r, z, t) ; \quad v_{i}=v_{i}(r, z, t) .
$$

Moreover, it is easily seen from the natural symmetry of the problem that

$$
v_{2}=0 ; \quad \sigma_{12}=\sigma_{23}=0
$$

in the cylindrical coordinate system. ${ }^{8}$ The first of the relations (4.4) expresses the fact that the flow must take place in planes through the $z$ axis, i.e. the axis of the bar. This condition can also be expressed by writing $v^{2}=0$, as we immediately see when we apply the process of raising or lowering the index of the velocity vector. Thus from (2.2) we have

$$
v_{1}=g_{1 k} v^{k}=v^{1} ; \quad v_{2}=g_{2 k} v^{k}=r^{2} v^{2} ; \quad v_{3}=g_{3 k} v^{k}=v^{3} .
$$

We shall first consider the case for which the surface $\Sigma$ is a fixed plane perpendicular to the axis of the bar (see \$1), and for definiteness we assume $\Sigma$ to be the plane $z=0$. For the parametric equations (3.1) we may now take

$$
r=u^{1} ; \quad \theta=u^{2} ; \quad z=0 \text {. }
$$

The components $g_{\alpha \beta}$ and $g^{\alpha \beta}$ of the metric tensor of this surface are given by

$$
\left\|g_{\alpha \beta}\right\|=\left\|\begin{array}{cc}
1 & 0 \\
0 & r^{2}
\end{array}\right\| ; \quad\left\|g^{\alpha \beta}\right\|=\left\|\begin{array}{cc}
1 & 0 \\
0 & \frac{1}{r^{2}}
\end{array}\right\|,
$$

${ }^{8}$ Cp. S. Timoshenko, Theory of Elasticity, McGraw-Hill, New York, 1934, p. 309. 
with individual components $g_{\alpha \beta}$ and $g^{\alpha \beta}$ equal to the elements in the $\alpha^{\text {th }}$ row and $\beta^{\text {th }}$ column of these matrices. Hence $g_{i j}=g_{\alpha \beta}$ and $g^{i i}=g^{\alpha \beta}$ if $i=\alpha$ and $j=\beta$ for this surface.

It will be assumed (see $\$ 1$ ) that the material in the bar is elastic and at rest within the region $z \geqq 0$. Let us now denote by $\bar{v}_{i}$ and $\bar{v}_{i, j}$ the values of the components $v_{i}$ of the velocity and the components $v_{i, j}$ of its covariant derivative on the plastic side of $\Sigma$. Obviously $\bar{v}_{3}=0$ for this problem, and since $\bar{v}_{2}=0$ from (4.4) the formula for the components $\bar{v}_{i, i}$ becomes

$$
\bar{v}_{i, j}=\frac{\partial \bar{v}_{i}}{\partial x^{j}}-\bar{v}_{1} \Gamma_{i j}^{1}
$$

where the bar on the first term in the right member denotes evaluation of the partial derivative on the plastic side of $\Sigma$. Taking account of (4.2) and the conditions on the $v$ 's given by (4.3) and (4.4), the above formula (4.6) now shows that

$$
\left\{\begin{array}{lll}
\bar{v}_{1,1}=\frac{\partial \bar{v}_{1}}{\partial r} ; & \bar{v}_{1,2}=0 ; & \bar{v}_{1,3}=\frac{\partial \bar{v}_{1}}{\partial z} \\
\bar{v}_{2,1}=0 ; & \bar{v}_{2,2}=r \bar{v}_{1} ; & \bar{v}_{2,3}=0 \\
\bar{v}_{3,1}=0 ; & \bar{v}_{3,2}=0 ; & \bar{v}_{3,3}=\frac{\partial \bar{v}_{3}}{\partial z}
\end{array}\right.
$$

Evidently the quantity $\partial \bar{v}_{1} / \partial r$ in these relations, which we defined as the derivative $\partial v_{1} / \partial r$ on the plastic side of $\Sigma$, can also be considered to be the partial derivative of $\bar{v}_{1}$ with respect to $r$.

The covariant derivative of the velocity vector $v$, considered as a vector on the surface $\Sigma$, will also enter into our discussion. Now the components $\bar{v}_{i ; \alpha}$ of this covariant derivative are given by the general formula

$$
\bar{v}_{i ; \alpha}=\frac{\partial \bar{v}_{i}}{\partial u^{\alpha}}-\bar{v}_{m} \Gamma_{i k}^{m} x_{\alpha}^{k},
$$

in which the $\bar{v}_{i}$ are functions of the parametric coordinates $u^{1}, u^{2}$ and the quantities $x_{\alpha}^{k}$ are derived from (4.5). Taking $i=1,2,3$ respectively we see immediately that the relations (4.8) reduce to the following:

$$
\bar{v}_{1 ; \alpha}=\frac{\partial \bar{v}_{1}}{\partial u^{\alpha}} ; \quad \bar{v}_{2 ; \alpha}=r \bar{v}_{1} x_{\alpha}^{2} ; \quad \bar{v}_{3 ; \alpha}=\frac{\partial \bar{v}_{3}}{\partial u^{\alpha}} .
$$

Hence we have

$$
\begin{cases}\bar{v}_{1 ; 1}=\frac{\partial \bar{v}_{1}}{\partial r} ; & \bar{v}_{1 ; 2}=0, \\ \bar{v}_{2 ; 1}=0 ; & \bar{v}_{2 ; 2}=r \bar{v}_{1} \\ \bar{v}_{3 ; 1}=0 ; & \bar{v}_{3 ; 2}=0 .\end{cases}
$$


5. Reduction of the compatibility conditions for the velocity. We may put

$$
\left[v_{i, i}\right]=\bar{v}_{i, j} ; \quad\left[\bar{v}_{i}\right]_{; \alpha}=\bar{v}_{i ; \alpha},
$$

since the velocity vanishes in the elastic region by hypothesis. Hence when $Z$ stands for the components $v_{i}$ of the velocity vector the geometrical conditions of compatibility (3.3) become

$$
\bar{v}_{i, i}=\lambda_{i} \nu_{i}+g^{\alpha \beta} g_{j k} \bar{v}_{i ; \alpha} x_{\beta}^{k},
$$

where the $\lambda$ 's are suitable functions defined on the surface $\Sigma$. But from the equation $z=0$, or the parametric equations (4.5) of the surface $\Sigma$, we have $\nu_{1}=\nu_{2}=0, \nu_{3}=1$ and $x_{\beta}^{k}=\delta_{\beta}^{k}$ for $k=1,2,3$ and $\beta=1,2$. Using (4.7) and (4.9) we now see immediately that the relations (5.1) reduce to identities when $j=1,2$. However, taking $j=3$ and $i=1,2,3$ the conditions (5.1) give

$$
\lambda_{1}=\frac{\partial \bar{v}_{1}}{\partial z} ; \quad \lambda_{2}=0 ; \quad \lambda_{3}=\frac{\partial \bar{v}_{3}}{\partial z} .
$$

6. Application of compatibility conditions. Evaluating (2.5) on the plastic side of $\Sigma$ and using the relations (4.7) and (5.2), we find that

$$
\lambda_{3}=-\left(\frac{\partial \bar{v}_{1}}{\partial r}+\frac{\bar{v}_{1}}{r}\right) \text {. }
$$

The equation (2.4) can be treated in a similar manner but the condition obtained will not be of use in this discussion. However, the equations (2.3) and (2.6) will yield significant conditions.

Assuming that a uniform tension prevails in the elastic region $(z \geqq 0)$, the stress components $\sigma_{i i}$ will have the values

$$
\sigma_{33}=\tau ; \quad \sigma_{i j}=0 \text { otherwise, }
$$

in this region, where $\tau>0$ denotes the constant tension over the ends of the bar. Let us furthermore assume, in conformity with the viewpoint stated in $\$ 1$, that the components $\sigma_{i j}$ and their first partial derivatives, and hence the components $\sigma_{i j, k}$ of the covariant derivative of the stress tensor, are continuous across the plane $\Sigma$. Then the values of the stress components $\sigma_{i j}$ on the plastic side of $\Sigma$ are also given by (6.2). Moreover, it follows that the components $\sigma_{i j, k}$ of the covariant derivative of the stress vanish on the plastic side of $\Sigma$ since these components must vanish in the elastic region on account of the condition of uniform stress. Hence when we evaluate (2.3) and (2.6) on the plastic side of $\Sigma$ we obtain

$$
\frac{\partial \bar{v}_{i}}{\partial t}+\bar{v}_{i, j} \bar{v}^{i}=0 ; \quad \bar{\epsilon}_{i j}=\bar{\psi} \sigma_{i j}^{*},
$$

where the bar is used to denote this evaluation as in the foregoing discussion and the $\sigma_{i j}^{*}$ are obtained from (6.2) in accordance with the equations (2.9). 
From (4.7) and the fact that $\bar{v}_{2}=\bar{v}_{3}=0$ we see that the first relation (6.3) is satisfied identically for $i=2,3$. But for $i=1$ this relation yields

$$
\frac{\partial \bar{v}_{1}}{\partial t}+\bar{v}_{1} \frac{\partial \bar{v}_{1}}{\partial r}=0
$$
by

Now from (2.8) and (4.7) we find that the values of the quantities $\bar{\epsilon}_{i j}$ are given

$$
\left\{\begin{array}{lll}
\bar{\epsilon}_{11}=\frac{\partial \bar{v}_{1}}{\partial r} ; & \bar{\epsilon}_{12}=0 ; & \bar{\epsilon}_{13}=\frac{1}{2} \frac{\partial \bar{v}_{1}}{\partial z}, \\
\bar{\epsilon}_{22}=r \bar{v}_{1} ; & \bar{\epsilon}_{23}=0 ; & \bar{\epsilon}_{33}=\frac{\partial \bar{v}_{3}}{\partial z} .
\end{array}\right.
$$

Also from (2.9) and (6.2) we have

$$
\left\{\begin{array}{lll}
\sigma_{11}^{*}=-\frac{1}{3} \tau ; & \sigma_{12}^{*}=0 ; & \sigma_{13}^{*}=0, \\
\sigma_{22}^{*}=-\frac{1}{3} \tau r^{2} ; & \sigma_{23}^{*}=0 ; & \sigma_{33}^{*}=\frac{2}{3} \tau .
\end{array}\right.
$$

Finally, it follows readily that

$$
\psi=\frac{1}{K} g^{i k} g^{i m} \sigma_{i j}^{*} \epsilon_{k m}=\frac{1}{K} g^{i k} g^{i m} \sigma_{i,} \epsilon_{k m},
$$

from (2.5), (2.8) and (2.9), i.e. the quantities $\sigma_{i j}^{*}$ can be replaced by $\sigma_{i j}$ in the

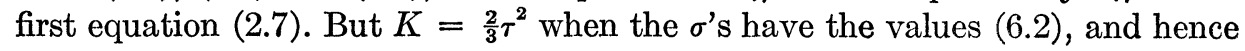
from (6.7) we obtain

$$
\bar{\psi}=\frac{3}{2 \tau} \frac{\partial \bar{v}_{3}}{\partial z} .
$$

Making the substitutions (6.5), (6.6) and (6.8), we find that three of the equations in the second set of equations (6.3) are satisfied identically. The remaining three equations become

$$
\begin{gathered}
2 \frac{\partial \bar{v}_{1}}{\partial r}+\frac{\partial \bar{v}_{3}}{\partial z}=0 \\
r \frac{\partial \bar{v}_{3}}{\partial z}+2 \bar{v}_{1}=0 \\
\frac{\partial \bar{v}_{1}}{\partial z}=0 .
\end{gathered}
$$

Hence $\lambda_{1}=0$ from (5.2) and (6.11). Also eliminating $\partial \bar{v}_{3} / \partial z$ between (6.9) and (6.10), we have

$$
\frac{\partial \bar{v}_{1}}{\partial r}=\frac{\bar{v}_{1}}{r}
$$

After the component $\bar{v}_{1}(r, t)$ has been found by integration of (6.4) and (6.12) 
we can determine $\partial \bar{v}_{3} / \partial z$ from (6.9) or (6.10). This determination automatically gives the value of $\lambda_{3}$ on account of the third equation (5.2).

7. Condition for rupture. Integrating (6.12) we have

$$
\bar{v}_{1}=C(t) r,
$$

where the coefficient $C$ depends on the time $t$ alone. Substituting the function $\bar{v}_{1}$ given by (7.1) into (6.4), we find that

$$
\frac{d C}{d t}+C^{2}=0
$$

But integration of (7.2) gives

$$
C=\frac{1}{t-A}
$$

where $A$ is a constant independent of the time. Hence from (7.1) and (7.3) we have

$$
\bar{v}_{1}=\frac{r}{t-A} \quad(t \geqq 0) .
$$

In the case of tension there will be a flow of plastic material toward the central axis, i.e. we shall have $\bar{v}_{1}<0$. But this implies $A>0$, as may be seen by taking $t=0$ in (7.4). Hence it follows from (7.4) that $\bar{v}_{1} \rightarrow-\infty$ as $t \rightarrow A$. The strength of the discontinuity on the plane $\Sigma$, as measured by $\left|\bar{v}_{1}\right|$, will therefore become infinite within a finite time $A$. Hence rupture will occur over the plane $\Sigma$ in accordance with the viewpoint expressed in $\$ 1$.

Remark 1. Excluding the case of unloading at the yield point for which $\psi<0$, we have $\psi \geqq 0$ in the plastic region. ${ }^{9}$ But $\bar{\psi}=0$ implies $\partial \bar{v}_{3} / \partial z=0$ from (6.8), and hence $\bar{v}_{1}=0$ on account of (6.10). This contradicts our assumption of a discontinuity in the velocity. Hence $\bar{\psi}>0$. Since $\tau>0$ for tension we now see that $\partial \bar{v}_{3} / \partial z>0$ from (6.8). Hence from (6.10) it follows that $\bar{v}_{1}<0$ as above assumed.

If unloading at the yield point occurs before the lapse of time required for rupture we can conclude, by an argument analogous to that employed in our foregoing paper, ${ }^{4}$ that rupture will fail to materialize over the plane $\Sigma$.

Remark 2. In the case of compression there will be a flow of material away from the central axis of the bar. Hence $\bar{v}_{1}>0$ and from this we see that the constant $A$ in (7.4) must be negative. Hence $\bar{v}_{1} \rightarrow 0$ as $t \rightarrow \infty$. In other words, any initial discontinuity $\bar{v}_{1}$ will be damped out and hence rupture will not occur over the plane $\Sigma$.

\footnotetext{
${ }^{9}$ See W. Prager \& P. G. Hodge, Jr., Theory of Perfectly Plastic Solids, J. Wiley and Sons, New York, 1951, p. 29; also R. HiLl, The Mathematical Theory of Plasticity, Oxford, Clarendon Press, 1950, p. 40.
} 
8. General symmetrical rupture. Let us now consider a surface of revolution about the axis of the bar as a possible surface of rupture $\Sigma$. In terms of the cylindrical coordinates $r, \theta, z$ such a surface will be given by an equation of the form

$$
F=z-f(r)=0,
$$

where $f$ is assumed to be an arbitrary differentiable function of the coordinate $r$. This surface can also be defined by writing

$$
y^{1}=r \cos \theta ; \quad y^{2}=r \sin \theta ; \quad y^{3}=f(r),
$$

in which $y^{1}, y^{2}, y^{3}$ are the rectangular coordinates introduced in $\$ 4$. We shall take the variables $u^{1}=r$ and $u^{2}=\theta$ to be the parametric coordinates of this surface $\Sigma$. Thus the equations (8.2) can be written

$$
y^{1}=u^{1} \cos u^{2} ; \quad y^{2}=u^{1} \sin u^{2} ; \quad y^{3}=f\left(u^{1}\right) .
$$

As in the foregoing discussion the cylindrical coordinates $r, \theta, z$ will also be denoted by $x^{1}, x^{2}, x^{3}$ respectively. The components $g_{\alpha \beta}$ of the metric tensor of the surface $\Sigma$ are given by the equations

$$
g_{\alpha \beta}=\frac{\partial y^{i}}{\partial u^{\alpha}} \frac{\partial y^{i}}{\partial u^{\beta}}
$$

in which the derivatives $\partial y^{i} / \partial u^{\alpha}$ are determined from (8.3). The values of the components $g_{\alpha \beta}$ and the contravariant components $g^{\alpha \beta}$, which are obtained from the $g_{\alpha \beta}$ in the usual manner, can be represented by writing

$$
\left\|g_{\alpha \beta}\right\|=\left\|\begin{array}{cc}
1+a^{2} & 0 \\
0 & r^{2}
\end{array}\right\| ; \quad\left\|g^{\alpha \beta}\right\|=\left\|\begin{array}{cc}
\frac{1}{1+a^{2}} & 0 \\
0 & \frac{1}{r^{2}}
\end{array}\right\|,
$$

where we have put

$$
a=\frac{d f(r)}{d r}
$$

The components of the metric tensor of the space, relative to the cylindrical system, are given by the matrices $\left\|g_{i j}\right\|$ and $\left\|g^{i j}\right\|$ in $\$ 4$. We shall continue to use Greek indices with the range 1, 2 for quantities associated with the surface $\Sigma$ and Latin indices with the range 1,2,3 for quantities associated with the space.

The covariant components $\nu_{i}$ of the unit normal vector to $\Sigma$ are now given by

$$
\nu_{i}=\frac{\partial F / \partial x^{i}}{\sqrt{g^{j k} \frac{\partial F}{\partial x^{j}} \cdot \frac{\partial F}{\partial x^{k}}}},
$$


where $F$ is defined by (8.1). Thus we find that

$$
\nu_{1}=\frac{-a}{\sqrt{1+a^{2}}} ; \quad \nu_{2}=0 ; \quad \nu_{3}=\frac{1}{\sqrt{1+a^{2}}} .
$$

This vector $\nu$ is assumed to be directed from the plastic into the elastic side of the surface $\Sigma$. From the relations

$$
\nu^{i}=g^{i j} \nu_{j}
$$

we have $\nu^{1}=\nu_{1}, \nu^{2}=0$ and $\nu^{3}=\nu_{3}$. Hence there is no difference in the values of the covariant and the contravariant components of this unit normal vector.

Denoting by $\bar{v}_{i}=\bar{v}_{i}(u, t)$ the values of the velocity components $v_{i}$ on the plastic side of $\Sigma$, let us consider the quantities

$$
\bar{v}_{i ; \alpha}=\frac{\partial \bar{v}_{i}}{\partial u^{\alpha}}-\bar{v}_{m} \Gamma_{i j}^{m} x_{\alpha}^{j},
$$

in which the $\Gamma$ 's are the Christoffel symbols defined in $\$ 4$ and the $x_{\alpha}^{i}$ are defined by (3.2). The values of these derivatives $x_{\alpha}^{i}$ are calculated from the equations

$$
x^{1}=u^{1} ; \quad x^{2}=u^{2} ; \quad x^{3}=f\left(u^{1}\right),
$$

which give the relations between the cylindrical coordinates $x$ and the parametric coordinates $u$ of the surface $\Sigma$. Taking cognizance of the fact that $\bar{v}_{2}=0$ from the symmetry condition (4.4), the explicit values of the components $\bar{v}_{i ; \alpha}$ are found to be

$$
\left\{\begin{array}{lll}
\bar{v}_{1 ; 1}=\frac{\partial \bar{v}_{1}}{\partial r} ; & \bar{v}_{2 ; 1}=0 ; & \bar{v}_{3 ; 1}=\frac{\partial \bar{v}_{3}}{\partial r}, \\
\bar{v}_{1 ; 2}=0 ; & \bar{v}_{2 ; 2}=r \bar{v}_{1} ; & \bar{v}_{3 ; 2}=0,
\end{array}\right.
$$

in which $u^{1}$ has been replaced by $r$, considered as a surface coordinate of $\Sigma$.

Let us next evaluate the components $v_{i, j}$ of the covariant derivative of $v$ on the plastic side of $\Sigma$. These components $\bar{v}_{i, j}$ are given by the formula

$$
\bar{v}_{i, i}=\frac{\partial \bar{v}_{i}}{\partial x^{j}}-\bar{v}_{m} \Gamma_{i j}^{m} .
$$

Here $\partial \bar{v}_{i} / \partial x^{i}$ denotes the value on $\Sigma$ of the partial derivative $\partial v_{i} / \partial x^{i}$ of $v_{i}$, considered as a function of the cylindrical coordinates $x$. Now if $\bar{v}_{i}$ denote the velocity components on the plastic side of $\Sigma$ we can write

$$
\frac{\partial \bar{v}_{i}}{\partial r}=\frac{\partial \bar{v}_{i}}{\partial x^{1}}+a \frac{\partial \bar{v}_{i}}{\partial x^{3}}=\frac{\partial \bar{v}_{i}}{\partial x^{1}}+a \frac{\partial \bar{v}_{i}}{\partial z},
$$

in which the left members are the derivatives of the $\bar{v}_{i}$ with respect to $r$, considered as a parametric coordinate of $\Sigma$, while the right members involve the derivatives $\partial v_{i} / \partial x^{1}$ and $\partial v_{i} / \partial x^{3}$ or $\partial v_{i} / \partial z$ on the plastic side of $\Sigma$. Calculating the quantities $\bar{v}_{i, j}$ by means of (8.6) and eliminating the derivatives $\partial \bar{v}_{i} / \partial x^{1}$ 
whenever these occur by the substitutions (8.7), we obtain

$$
\left\{\begin{array}{lll}
\bar{v}_{1,1}=\frac{\partial \bar{v}_{1}}{\partial r}-a \frac{\partial \bar{v}_{1}}{\partial z} ; & \bar{v}_{2,1}=0 ; & \bar{v}_{3,1}=\frac{\partial \bar{v}_{3}}{\partial r}-a \frac{\partial \bar{v}_{3}}{\partial z}, \\
\bar{v}_{1,2}=0 ; & \bar{v}_{2,2}=r \bar{v}_{1} ; & \bar{v}_{3,2}=0, \\
\bar{v}_{1,3}=\frac{\partial \bar{v}_{1}}{\partial z} ; & \bar{v}_{2,3}=0 ; & \bar{v}_{3,3}=\frac{\partial \bar{v}_{3}}{\partial z} .
\end{array}\right.
$$

Assuming that the material in the elastic region is at rest, the components $\bar{v}_{i ; \alpha}$ and $\bar{v}_{i, j}$ in (8.5) and (8.8) are related by the geometrical conditions of compatibility, namely

$$
\bar{v}_{i, j}=\lambda_{i} \nu_{i}+g^{\alpha \beta} g_{j k} \bar{v}_{i ; \alpha} x_{\beta}^{k},
$$

in which the $\lambda_{i}$ are functions on the surface $\Sigma$. Examination of the equations (8.9) yields the condition $\lambda_{2}=0$ and the following two relations:

$$
\left\{\begin{array}{l}
\lambda_{1}=\sqrt{1+a^{2}} \frac{\partial \bar{v}_{1}}{\partial z}-\frac{a}{\sqrt{1+a^{2}}} \frac{\partial \bar{v}_{1}}{\partial r}, \\
\lambda_{3}=\sqrt{1+a^{2}} \frac{\partial \bar{v}_{3}}{\partial z}-\frac{a}{\sqrt{1+a^{2}}} \frac{\partial \bar{v}_{3}}{\partial r} .
\end{array}\right.
$$

By total time differentiation of (8.1) we obtain

$$
\frac{d z}{d t}=a \frac{d r}{d t} .
$$

Hence

$$
\bar{v}^{3}=a \bar{v}^{1} ; \quad \text { or } \quad \bar{v}_{3}=a \bar{v}_{1},
$$

since, as we have already observed in $\S 4$, there is no difference in the values of corresponding covariant and contravariant components of the velocity vector.

As in our foregoing discussion we assume that the stress components $\sigma_{i i}$ have the values (6.2) in the elastic region; also that these components are continuous and have continuous first partial derivatives across the surface $\Sigma$. We thus arrive again at the relations (6.3). Substituting the values of the $\bar{v}_{i, j}$ given by (8.8), the first relation (6.3) is readily seen to be satisfied identically for $i=2$. However, for $i=1,3$ we obtain

$$
\begin{aligned}
& \frac{\partial \bar{v}_{1}}{\partial t}+\bar{v}_{1} \frac{\partial \bar{v}_{1}}{\partial r}=0 \\
& \frac{\partial \bar{v}_{3}}{\partial t}+\bar{v}_{1} \frac{\partial \bar{v}_{3}}{\partial r}=0
\end{aligned}
$$

when use is made of (8.10). 
Eliminating $\bar{v}_{3}$ from (8.12) by means of (8.10), we find that

$$
\left(\frac{\partial \bar{v}_{1}}{\partial t}+\bar{v}_{1} \frac{\partial \bar{v}_{1}}{\partial r}\right)+\frac{\bar{v}_{1}^{2}}{a} \frac{d a}{d r}=0 .
$$

Now if $\bar{v}_{1}=0$ we have $\bar{v}_{3}=0$ from (8.10) and hence $\bar{v}_{i}=0$ for $i=1,2,3$, which contradicts our assumption of a discontinuity in the velocity over the surface $\Sigma$. Hence $\bar{v}_{1} \neq 0$, and from (8.11) and (8.13) it therefore follows that the derivative $d a / d r$ vanishes. But this means that $f(r)=a r+b$ where $a$ and $b$ are constants. Hence the equation of the surface $\Sigma$ can be given the form $z=a r$ by a proper choice of the origin of coordinates. Thus we see that the surface $\Sigma$ must be a circular cone whose axis coincides with the axis of the bar.

Turning next to the second set of relations (6.3), we first note that

$$
\left\{\begin{array}{lll}
\bar{\epsilon}_{11}=\frac{\partial \bar{v}_{1}}{\partial r}-a \frac{\partial \bar{v}_{1}}{\partial z} ; & \bar{\epsilon}_{12}=0 ; & \bar{\epsilon}_{13}=\frac{1}{2}\left(\frac{\partial \bar{v}_{1}}{\partial z}+\frac{\partial \bar{v}_{3}}{\partial r}-a \frac{\partial \bar{v}_{3}}{\partial z}\right), \\
\bar{\epsilon}_{22}=r \bar{v}_{1} ; & \bar{\epsilon}_{23}=0 ; & \bar{\epsilon}_{33}=\frac{\partial \bar{v}_{3}}{\partial z},
\end{array}\right.
$$

as follows from (2.8) and (8.8). We also observe that the quantity $\bar{\psi}$ is again given by (6.8) and that the $\sigma_{i i}^{*}$ have the previous values (6.6). Using (6.6), (6.8) and (8.14) we now find that three of the equations in the second set of equations (6.3) are satisfied identically while the remaining equations can be written

$$
\begin{gathered}
\frac{\partial \bar{v}_{1}}{\partial r}-a \frac{\partial \bar{v}_{1}}{\partial z}=-\frac{1}{2} \frac{\partial \bar{v}_{3}}{\partial z} \\
\frac{\partial \bar{v}_{1}}{\partial z}+\frac{\partial \bar{v}_{3}}{\partial r}-a \frac{\partial \bar{v}_{3}}{\partial z}=0 \\
\frac{\partial \bar{v}_{3}}{\partial z}=-\frac{2 \bar{v}_{1}}{r}
\end{gathered}
$$

Now eliminate $\partial \bar{v}_{3} / \partial z$ from (8.15) and (8.16) by means of (8.17). This gives

$$
\begin{gathered}
\frac{\partial \bar{v}_{1}}{\partial r}-a \frac{\partial \bar{v}_{1}}{\partial z}=\frac{\bar{v}_{1}}{r}, \\
\frac{\partial \bar{v}_{1}}{\partial z}+\frac{\partial \bar{v}_{3}}{\partial r}+\frac{2 a \bar{v}_{1}}{r}=0 .
\end{gathered}
$$

Next, eliminating $\partial \bar{v}_{1} / \partial z$ between (8.18) and (8.19), we obtain

$$
\frac{\partial \bar{v}_{1}}{\partial r}+\left(2 a^{2}-1\right) \frac{\bar{v}_{1}}{r}+a \frac{\partial \bar{v}_{3}}{\partial r}=0 .
$$

But, since $a$ is a constant, it follows from (8.10) that

$$
\frac{\partial \bar{v}_{3}}{\partial r}=a \frac{\partial \bar{v}_{1}}{\partial r}
$$


Hence from (8.20) and (8.21) we have

$$
\frac{\partial \bar{v}_{1}}{\partial r}=A \frac{\bar{v}_{1}}{r}, \quad A=\frac{1-2 a^{2}}{1+a^{2}} .
$$

Case I. If $A \neq 0$ we can integrate (8.22) to obtain

$$
\bar{v}_{1}=C(t) r^{A},
$$

where the coefficient $C$ is a function of the time $t$. Substituting this expression for $\bar{v}_{1}$ into (8.11), the resulting equation can be written

$$
\frac{1}{C^{2}} \frac{d C}{d t}=-\frac{A r^{A}}{r}
$$

But the left member of this relation depends at most on the time $t$ and the right member at most on the coordinate $r$. Each member of the relation must therefore reduce to a constant since the variables $r$ and $t$ are mutually independent. Hence we must have

$$
A=\frac{1-2 a^{2}}{1+a^{2}}=1
$$

It follows that $a=0$. Hence $\Sigma$ must be a plane perpendicular to the axis of the bar. This case has been discussed in $\$ 4, \cdots, \S 7$ and, as we have seen, leads to rupture of the bar over a plane perpendicular to its axis.

Case II. If $A=0$ it follows that $a^{2}=\frac{1}{2}$, and hence $\Sigma$ is a circular cone $C$ whose generators make an angle of $35^{\circ} 16^{\prime}$ with a plane perpendicular to the axis of the bar. Also $\partial \bar{v}_{1} / \partial r=0$ from (8.22) and hence $\partial \bar{v}_{3} / \partial r=0$ from (8.21). Moreover, it follows from the equations (8.11) and (8.13) that the partial time derivatives of $\bar{v}_{1}$ and $\bar{v}_{3}$ must vanish. Thus we see that the magnitude of the velocity vector $\bar{v}_{1}$, which is obviously directed along the generators of the cone $C$, is constant both with respect to position and time.

Remark 1. In the discussion of the Lüders band problem we proved a result concerning the velocity similar to that in the above italicized statement. It was also shown that a discontinuity in the first derivatives of the velocity components must become infinite within a finite time. Corresponding behavior of the first derivatives must therefore be expected for the case of the above circular cone $C$, due to the close analogy between this case and the Lüders band problem. Considering the discontinuity in these derivatives as well as the discontinuity in the velocity itself, we would therefore arrive at the strict condition for fracture over the conical surface $C$ according to the standards of $\$ 1$. However, the demonstration of this result will require the use of compatibility conditions of the second order, and it will therefore not be given in this paper.

Remark 2. Comparison of the singularities over the circular cone $C$ and the plane $\Sigma$ indicates that a stronger instability will occur in the material over 
the latter surface. This observation would lead us to expect that fracture will actually begin within the plane $\Sigma$ or central portion of the "cup and cone" type of rupture.

Remark 3. Significant deviations from the stress field assumed in the above discussion may possibly occur in the case of ductile materials at points of the necked region lying off the minimum section $P Q$ in Fig. 4. The use of a closer approximation to this stress field might lead to a certain modification of the result on conical fracture.

9. Rupture in plastic region of neck. Let us now consider the more realistic situation in which the bar necks down as indicated in Fig. 4 and plastic flow occurs in the region containing the minimum section of the neck $P Q$. Assume that a small discontinuity in the velocity develops across the plane $P Q$, which will hereafter be denoted by the single letter $\Sigma$, in conformity with our previous notation. We shall investigate the question of whether this discontinuity will be damped out or will increase indefinitely so as to yield a surface of strong instability or rupture.

As in our previous discussion we can introduce the rectangular coordinate system $y$ and the cylindrical coordinates $r, \theta, z$ which we shall also denote by $x^{1}, x^{2}, x^{3}$ respectively. Taking the variables $u^{1}=r$ and $u^{2}=\theta$ to be the parametric coordinates of the plane $\Sigma$, the components of the metric tensors for the space and for the plane $\Sigma$ will be given by the proper metrices in $\$ 4$. Also the specific symmetry relations (4.3) and (4.4) will be valid.

Since the plane $\Sigma$ is stationary in this discussion we shall have the relation

$$
\rho v_{n}=\bar{\rho} \bar{v}_{n}
$$

where $\rho$ and $v_{n}$ denote the density and normal velocity on one side of $\Sigma$, while $\bar{\rho}$ and $\bar{v}_{n}$ are the corresponding quantities on the other side of this plane. We assume that the density $\rho$ is continuous across the plane $\Sigma$ (see \$1). Hence $v_{n}=\bar{v}_{n}$ from the above relation or, in other words, the discontinuity $\left[v_{3}\right]$ must vanish. Actually, we are justified in assuming from symmetry that the component $v_{3}$, or the numerically equal component $v^{3}$, vanishes on each side of the plane $\Sigma$. Accordingly, this assumption will be made and used in the following discussion. Now $\left[v_{2}\right]$ must also vanish from the symmetry condition (4.4). Hence we must have $\left[v_{1}\right] \neq 0$ since we have assumed above that a discontinuity in the velocity occurs across the plane $\Sigma$.

From the general conditions of compatibility (3.3) we now obtain

$$
\left[v_{i, j}\right]=\lambda_{i} \nu_{j}+g^{\alpha \beta} g_{i k}\left[v_{i}\right]_{; \alpha} x_{\beta}^{k},
$$

in which the various terms have their previous significance, and

$$
\left[v_{i}\right]_{; \alpha}=\frac{\partial\left[v_{i}\right]}{\partial u^{\alpha}}-\left[v_{m}\right] \Gamma_{i k}^{m} x_{\alpha}^{k},
$$




$$
\left[v_{i, j}\right]=\left[\frac{\partial v_{i}}{\partial x^{j}}\right]-\left[v_{m}\right] \Gamma_{i j}^{m} .
$$

From (9.2) and (9.3) we now find that

$$
\left\{\begin{array}{lll}
{\left[v_{1}\right]_{; 1}=\frac{\partial\left[v_{1}\right]}{\partial r} ;} & {\left[v_{2}\right]_{; 1}=0 ;} & {\left[v_{3}\right]_{; 1}=0} \\
{\left[v_{1}\right]_{; 2}=0 ;} & {\left[v_{2}\right]_{; 2}=r\left[v_{1}\right] ;} & {\left[v_{3}\right]_{; 2}=0,}
\end{array}\right.
$$

and

$$
\left\{\begin{array}{lll}
{\left[v_{1,1}\right]=\frac{\partial\left[v_{1}\right]}{\partial r} ;} & {\left[v_{2,1}\right]=0 ;} & {\left[v_{3,1}\right]=0,} \\
{\left[v_{1,2}\right]=0 ;} & {\left[v_{2,2}\right]=r\left[v_{1}\right] ;} & {\left[v_{3,2}\right]=0,} \\
{\left[v_{1,3}\right]=\frac{\partial\left[v_{1}\right]}{\partial z} ;} & {\left[v_{2,3}\right]=0 ;} & {\left[v_{3,3}\right]=\left[\frac{\partial v_{3}}{\partial z}\right] .}
\end{array}\right.
$$

But from (9.1) we have

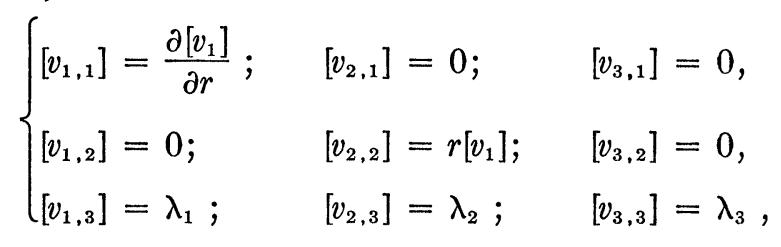

when use is made of (9.4). Hence, equating the right members of corresponding relations in (9.5) and (9.6), it follows that

$$
\lambda_{1}=\left[\frac{\partial v_{1}}{\partial z}\right] ; \quad \lambda_{2}=0 ; \quad \lambda_{3}=\left[\frac{\partial v_{3}}{\partial z}\right]
$$

From the equation of incompressibility (2.5) we obtain

$$
g^{i j}\left[v_{i, j}\right]=\left[v_{1,1}\right]+\frac{1}{r^{2}}\left[v_{2,2}\right]+\left[v_{3,3}\right]=0 .
$$

Using (9.6) the condition (9.8) can be written

$$
\lambda_{3}=-\left(\frac{\partial\left[v_{1}\right]}{\partial r}+\frac{\left[v_{1}\right]}{r}\right)
$$

We shall again make the assumption that the $\sigma_{i j}$ and their first partial derivatives are continuous across the surface $\Sigma$. Beyond this condition we shall consider, for the present, that the $\sigma$ 's are subjected only to the symmetry requirement in (4.3) and (4.4). From (2.3) and (2.6) we therefore obtain

$$
\begin{gathered}
\frac{\partial\left[v_{i}\right]}{\partial t}+\left[v_{i, i} v^{j}\right]=0, \\
{\left[\frac{d \sigma_{i j}^{*}}{d t}\right]=2 \mu\left(\left[\epsilon_{i j}\right]-[\psi] \sigma_{i j}^{*}\right) .}
\end{gathered}
$$


The term $\left[v_{i, i} v^{i}\right]$ in (9.10) reduces to $\left[v_{i, 1} v^{1}\right]$ from the symmetry condition (4.4) and the above assumption that the component $v^{3}$ vanishes on each side of the plane $\Sigma$. This term can also be written $\left[v_{i, 1} v_{1}\right]$ since the quantities $v_{1}$ and $v^{1}$ have equal values. Hence (9.10) becomes

$$
\frac{\partial\left[v_{i}\right]}{\partial t}+\left[v_{i, 1} v_{1}\right]=0 .
$$

But $v_{2,1}$ and $v_{3,1}$ vanish on each side of $\Sigma$; this follows readily from the formula for the covariant derivative of the velocity vector. For $i=2,3$ the equation (9.12) is therefore satisfied identically. However, $v_{1,1}=\partial v_{1} / \partial r$ on each side of $\Sigma$ and hence, for $i=1$, the equation (9.12) becomes

$$
\frac{\partial\left[v_{1}\right]}{\partial t}+\frac{1}{2} \frac{\partial\left[v_{1}^{2}\right]}{\partial r}=0 .
$$

Taking account of the continuity of the $\sigma$ 's across $\Sigma$ and the vanishing of the discontinuities $\left[v^{2}\right]$ and $\left[v^{3}\right]$, we observe that

$$
\left[\frac{d \sigma_{i j}^{*}}{d t}\right]=\frac{\partial}{\partial t}\left[\sigma_{i j}^{*}\right]+\sigma_{i i, k}^{*}\left[v^{k}\right]=\sigma_{i, j 1}^{*}\left[v_{1}^{1}\right] .
$$

Hence (9.11) can be written

$$
\left[\epsilon_{i j}\right]-\sigma_{i i}^{*}[\psi]=\sigma_{i i, 1}^{*} \frac{\left[v_{1}\right]}{2 \mu},
$$

since $v_{1}=v^{1}$. Now consider the two relations

$$
\left\{\begin{array}{l}
{\left[\epsilon_{11}\right]-\sigma_{11}^{*}[\psi]=\sigma_{11,1}^{*} \frac{\left[v_{1}\right]}{2 \mu},} \\
{\left[\epsilon_{22}\right]-\sigma_{22}^{*}[\psi]=\sigma_{22,1}^{*} \frac{\left[v_{1}\right]}{2 \mu},}
\end{array}\right.
$$

which we obtain by taking $i, j=1,1$ and $i, j=2,2$ in (9.15). But

$$
\left[\epsilon_{11}\right]=\frac{\partial\left[v_{1}\right]}{\partial r} ; \quad\left[\epsilon_{22}\right]=r\left[v_{1}\right] .
$$

Making these substitutions and eliminating the quantity $[\psi]$ from the above two relations we have

$$
\frac{\partial\left[v_{1}\right]}{\partial r}=\left(\frac{\sigma_{11}^{*} r}{\sigma_{22}^{*}}+\frac{\sigma_{11,1}^{*}}{2 \mu}-\frac{\sigma_{11}^{*}}{\sigma_{22}^{*}} \frac{\sigma_{22,1}^{*}}{2 \mu}\right)\left[v_{1}\right] .
$$

It would appear that the values (6.2), in which $\tau$ is the yield stress, furnish a first approximation for the stress components $\sigma_{i j}$ over the minimum section $P Q$ in Fig. 4 , i.e. over the plane $\Sigma$, although significant deviations from these values may possibly occur at other points within the plastic region of the neck. Assuming that the $\sigma_{i i}$ can be approximated by equations of the form (6.2) over the plane $\Sigma$, it follows from the formula for the covariant derivative of the stress deviator $\sigma^{*}$ that the components $\sigma_{11,1}^{*}$ and $\sigma_{22,1}^{*}$ in (9.16) are equal to 
zero; moreover, we have

$$
\frac{\sigma_{11}^{*} r}{\sigma_{22}^{*}}=\frac{1}{r}
$$

from (6.6). Hence (9.16) reduces to

$$
\frac{\partial\left[v_{1}\right]}{\partial r}=\frac{\left[v_{1}\right]}{r}
$$

Integrating (9.17) we obtain

$$
\left[v_{1}\right]=C(t) r,
$$

in which the coefficient $C$ depends at most on the time $t$. It is evident from (9.18) that the function $C(t)$ will have a continuous derivative with respect to $t$ since we assume the velocity components $v_{i}(x, t)$ to be continuously differentiable functions.

Let us now consider the discontinuity $\left[v_{1}\right]=\bar{v}-v$, where we denote by $\bar{v}$ and $v$ the values of the component $v_{1}$ at contiguous points on the two sides of $\Sigma$. Then we readily see that

$$
\left[v_{1}^{2}\right]=\bar{v}^{2}-v^{2}=\left[v_{1}\right]^{2}+2 v\left[v_{1}\right],
$$

or, making the substitution (9.18), we have

$$
\left[v_{1}^{2}\right]=C^{2} r^{2}+2 C v r .
$$

Hence from (9.18) and (9.19) the equation (9.13) becomes

$$
\frac{1}{C} \frac{d C}{d t}+C=-\left(\frac{\partial v}{\partial r}+\frac{v}{r}\right)
$$

But the left member of (9.20) can depend at most on the time $t$. Hence (9.20) can be replaced by the equivalent system of equations

$$
\begin{gathered}
\frac{1}{C} \frac{d C}{d t}+C=2 E(t), \\
\frac{\partial v}{\partial r}+\frac{v}{r}=-2 E(t)
\end{gathered}
$$

where $E$ depends only on the time $t$.

Assuming $v \neq \infty$ at $r=0$, we have

$$
v=-E(t) r
$$

by integration of (9.22). Now for the tension problem under consideration the flow is obviously toward the central axis of the bar. Hence we must have $v<0$. It follows therefore from (9.23) that

$$
E(t)>0 \text {. }
$$


Also, integrating (9.21), we obtain

$$
C(t)=\frac{\exp \left\{2 \int_{0}^{t} E(t) d t\right\}}{\int_{0}^{t} \exp \left\{2 \int_{0}^{t} E(t) d t\right\} d t+\frac{1}{C(0)}},
$$

where the quantity $C(0)$ in the right member of this equation denotes the value of the function $C(t)$ at time $t=0$.

It is natural to assume that the initial discontinuity $\left[v_{1}\right]$, i.e. the value of $\left[v_{1}\right]$ at time $t=0$, is the result of a slip of the material particles on one side of the plane $\Sigma$ toward the central axis of the bar. For definiteness let us suppose that this slip occurs on the side of $\Sigma$ for which the velocity has been denoted by $\bar{v}$. But this means that $|\bar{v}|>|v|$ and hence $\left[v_{1}\right]<0$ at $t=0$. Hence from (9.18) the initial value of the function $C(t)$ must be negative, i.e. $C(0)<0$. But since $E(t)$ is positive from (9.24) it will always be possible to find a positive number $A$ such that

$$
\int_{0}^{A} \exp \left\{2 \int_{0}^{t} E(t) d t\right\} d t+\frac{1}{C(0)}=0 .
$$

Hence from (9.25) it follows that $C(t) \rightarrow-\infty$ as $t \rightarrow A>0$. In other words, the initial discontinuity $\left[v_{1}\right]$ must become indefinitely large within a finite time. The plane $\Sigma$ is therefore a surface of strong instability over which the bar is subject to rupture, in accordance with the viewpoint of $\$ 1$.

Graduate Institute for Mathematics and Mechanics

Bloomington, Indiana 